\title{
Analysis of the Seasonal Variation in Chemical Profile of Piper glabratum Kunth Essential Oils Using GC×GC/qMS and Their Antioxidant and Antifungal Activities
}

\author{
Anaí L. dos Santos, ${ }^{a}$ Allan S. Polidoro, ${ }^{a}$ Cláudia A. L. Cardoso, ${ }^{b}$ Jonas S. Mota, ${ }^{b}$ \\ Rosângela A. Jacques ${ }^{a}$ and Elina B. Caramão ${ }^{\circledR *, a, c, d}$ \\ anstituto de Química, Universidade Federal do Rio Grande do Sul (UFRGS), \\ 91509-900 Porto Alegre-RS, Brazil \\ ${ }^{b}$ Centro de Estudos em Recursos Naturais, Universidade Estadual do Mato Grosso do Sul (UEMS), \\ 79804-970 Dourados-MS, Brazil \\ 'Programa de Pós-Graduação em Biotecnologia Industrial, Universidade Tiradentes (UNIT), \\ 49032-490 Aracaju-SE, Brazil \\ ${ }^{d}$ Instituto Nacional de Ciências e Tecnologia-Energia e Meio Ambiente (INCT E\&A), \\ 40170-115 Salvador-BA, Brazil
}

\begin{abstract}
Piper glabratum is a plant native from South America, which has been little studied, despite its use in folk medicine. In this study, chemical composition, antioxidant and antifungal activities of essential oils (EOs) from leaves of $P$. glabratum were investigated. The influence of seasonality on its chemical composition was also evaluated. The chemical characterization was performed by twodimensional gas chromatography coupled with quadrupole mass spectrometry (GC×GC/qMS). Altogether, 199 compounds were identified in the EOs and their composition varied during the seasonal cycle. The main compounds found were sesquiterpenes and their oxygenated analogues. EOs showed antioxidant activity, measured by 2,2-diphenyl-1-picrylhydrazyl-DPPH (from 28.1 to $33.4 \mu \mathrm{g} \mathrm{mL}^{-1}$ ) and $\beta$-carotene assays (from 57.4 to $66.4 \%$ ), and inhibitory action against $C$. albicans. The oils provided expressive properties; however, the yield of EO from winter was not enough for activities assays, remarking the influence of the seasonality on the biological activities of this plant.
\end{abstract} activity

Keywords: essential oil, Piper glabratum, GC $\times$ GC/qMS, antifungal activity, antioxidant

\section{Introduction}

Essential oils are complex mixtures of compounds which vary at different concentrations with commercially important especially for the pharmaceutical, food, sanitary, and cosmetic industries. The main compounds of these oils are terpenes and terpenoids and the other aromatic and aliphatic constituents, all characterized by relatively low molecular weight. ${ }^{1}$ Interactions between these compounds may lead to antagonistic, additive, or synergistic effects, inducing higher or lower bioactivity when compared to the oil's isolated components. ${ }^{2}$

The genus Piper contains over 700 species, distributed in tropical and subtropical region of the world. ${ }^{3}$ Many species,

*e-mail: elina@ufrgs.br mainly from India, Asia, and Africa, are economically important; they have been used for centuries in folk medicine, as well as a food additive. ${ }^{4}$ The essential oils from different Piper species commonly found in Brazil have been studied, and have showed a rich chemical composition, as well as biocidal, cytotoxic, antifungal, antioxidant, and anti-cholinesterase activities. ${ }^{5-7}$ Among the Piper species native from Brazil, Piper glabratum Kunth has been little studied, despite its long use in folk medicine, and the studies that have pointed to its prominent diuretic proprieties, ${ }^{8}$ as well as its antiparasitic activity against Leishmania and Trypanosoma cruzi. ${ }^{9}$ Additionally, it has been showed that the essential oils possess anti-inflammatory potential and absence of toxicity. ${ }^{10}$

Comprehensive two-dimensional gas chromatography $(\mathrm{GC} \times \mathrm{GC})^{11}$ is now the most powerful separation technique 
for the analysis of volatile compounds. ${ }^{12}$ Since the first application of $\mathrm{GC} \times \mathrm{GC}$ for essential oil characterization, ${ }^{13}$ much attention has been devoted to this field, due to its superiority over one-dimensional gas chromatography (1D-GC) for analysis of this matrix.

In $\mathrm{GC} \times \mathrm{GC}$, fast detectors with high data acquisition rate are mandatory. This feature is required for the correct assignment and quantification of the very fast second-dimension peaks (100-600 ms). A relation exists between the acquisition rate and the number of data points which define a peak. The higher the acquisition rate, the greater the number of data points that is available to define the chromatographic profile. ${ }^{14,15}$ Furthermore, there is a compromise between the identification of compounds with a high level of confidence by mass spectrometry, and the mass spectra quality generated by these systems. It can be studied by evaluating spectral skewing and similarity match (mass spectral similarity, \%MS), with the MS library compounds, at each data point of the modulated peak. Scanning of fast-eluting peaks can cause skewing of the mass spectra, i.e., variation of the instantaneous mass flux in the ion source over a single scan cycle and can be described by plotting mass-to-charge ratio $(\mathrm{m} / \mathrm{z})$ of abundant ions in a spectrum across the peak elution. ${ }^{16}$

The goal of the present study was to investigate the chemical profile and seasonal variation of the essential oils from Piper glabratum Kunth by rapid-scanning two-dimensional gas chromatography coupled with quadrupole mass spectrometry (GC×GC/qMS). Initially, the detector's performance (in terms of number of data points per peak, average spectra quality match, and peak skewing) was evaluated against representative essential oil compounds. The antioxidant and antifungal activity of the oils were also evaluated.

\section{Experimental}

\section{Samples and reagents}

The leaves of Piper glabratum were collected during 2012, according the four stations: March (autumn), June (winter), September (spring) and December (summer), in Dourados-MS (22 ${ }^{\circ} 14^{\prime} 55^{\prime \prime} S$; 54 53'57'W), Brazil. A voucher specimen was identified (DDMS 4412) by Dra Elsie Franklin Guimarães and deposited in the herbarium of the Federal University of Grande Dourados (UFGD).

Standard and chemicals compounds: $n$-alkane $\left(\mathrm{C}_{6}-\mathrm{C}_{30}\right)$, $\beta$-carotene ( $\geq 95 \%)$, linoleic acid ( $\geq 98 \%$ ), 2,2-diphenyl1-picrylhydrazyl (DPPH), nystatin, and butylated hydroxytoluene (BHT) ( $\geq 99 \%)$, were purchased from Sigma Aldrich (Saint Louis, MO, USA). Solutions of $n$-alkane (100 ppm) were prepared in $n$-hexane. Sabouraud broth and polyoxyethylene sorbitan monopalmitate (Tween ${ }^{\circledR}-40$ ) were purchased from Sigma Aldrich (Saint Louis, MO, USA), and anhydrous sodium sulfate p.a. grade from Merck (Darmstadt, HE, Germany). Methanol, chloroform and $n$-hexane (chromatographic grade) were obtained from JT Baker (Phillipsburg, NJ, USA).

Essential oil standards: (-)- $\beta$-pinene (99\%), $\alpha$-terpineol ( $\geq 96 \%$ ), limonene (97\%), linalool (97\%), (E)-caryophyllene ( $\geq 98.5 \%)$, carvone $(\geq 97 \%)$, $\alpha$-humulene $(\geq 96 \%)$, 1,8-cineole (99\%), camphor ( $\geq 96 \%)$, $(E)$-nerolidol $(\geq 85 \%)$ and $\alpha$-bisabolol $(\geq 93 \%)$ were purchased from Sigma Aldrich (Saint Louis, MO, USA). Standard solutions (1 ppm) were prepared by weight appropriate amounts of standard, dilution in hexane and stored at $-4{ }^{\circ} \mathrm{C}$.

\section{Sample preparation}

The leaves were collected, chopped, and immediately hydro-distilled for $4 \mathrm{~h}$ using a Clevenger-type apparatus. The essential oil was dried using anhydrous sodium sulfate, transferred to dark vials, weight, and stored at $-4{ }^{\circ} \mathrm{C}$ for further analysis. The mass yields (weight to weight) were calculated on fresh weight basis. Each essential oil $(50 \mathrm{mg})$ was diluted in $n$-hexane $(1 \mathrm{~mL})$ before gas chromatographic analysis. The experiments were made in triplicate.

\section{$\mathrm{GC} \times \mathrm{GC} / \mathrm{qMS}$ analysis}

GC $\times$ GC/qMS analysis was carried out on a Shimadzu GC $\times$ GC/qMS system consisting of a GC2010 gas chromatograph and a QP2010 Ultra quadrupole mass spectrometer (Shimadzu Corp., TYO, Japan). The mass spectrometer was operated at $70 \mathrm{eV}$ in fast scan mode (20000 Daltons per s) over a 40-250 $\mathrm{m} / \mathrm{z}$ range, giving a $65 \mathrm{~Hz}$ acquisition rate. The system was provided with a loop-type modulator (Zoex Corp., TX, USA) cooled with liquid nitrogen and with the hot jet pulse time set at $375 \mathrm{~ms}$ $\left(300{ }^{\circ} \mathrm{C}\right)$ with modulation times of $5 \mathrm{~s}$. The separations were achieved on a DB-5 (5\% phenyl methyl-polysiloxane, Agilent Technologies, CA, USA) analytical column $(60 \mathrm{~m} \times 0.25 \mathrm{~mm} \times 0.25 \mu \mathrm{m})$ as a first dimension (1D). The second dimension (2D) column was a DB-17 (50\% phenyl methyl-polysiloxane, Agilent Technologies, CA, USA) analytical column $(2.15 \mathrm{~m} \times 0.18 \mathrm{~mm} \times 0.18 \mu \mathrm{m})$. Temperatures were set as follows: $40^{\circ} \mathrm{C}$ (hold $5 \mathrm{~min}$ ), raised to $115^{\circ} \mathrm{C}\left(3{ }^{\circ} \mathrm{C} \mathrm{min}{ }^{-1}\right)$, and then to $175^{\circ} \mathrm{C}\left(2^{\circ} \mathrm{C} \mathrm{min}^{-1}\right)$ and finally $246{ }^{\circ} \mathrm{C}\left(3{ }^{\circ} \mathrm{C} \mathrm{min}{ }^{-1}\right)$ (hold $\left.10 \mathrm{~min}\right)$. The injector, ion source and interface temperature were held at $250^{\circ} \mathrm{C}$. The samples were injected in the split mode (1:20) and the carrier gas flow rate (helium) was $0.91 \mathrm{~mL} \mathrm{~min}^{-1}$. In 
order to calculate the linear-temperature-programmed retention indices (LTPRI) a linear $\mathrm{C}_{6}-\mathrm{C}_{30}$ alkane mixture was analyzed using identical GCxGC/qMS conditions. ${ }^{17}$ Data were acquired by GCMS Solution software ${ }^{18}$ and processed using GCImage software. ${ }^{19}$ Compounds were tentatively identified by comparing their electron ionization mass spectral fragmentation (EI-MS) similarity with commercial database (NIST) and by comparing their LTPRI to those reported in a database. ${ }^{20}$ An identity spectrum match factor above 800 resulting from NIST and a LTPRI with a match window of \pm 20 were determined to be acceptable for tentatively identified compound. The relative amounts of individual compounds were calculated based on $\mathrm{GC} \times \mathrm{GC} / \mathrm{qMS}$ peak area without using correction factor.

\section{Antioxidant activity}

The evaluation of the antioxidant capacity was carried out by two different methods: DPPH and $\beta$-carotene bleaching.

\section{DPPH: free radical scavenging assay}

In order to estimate the DPPH scavenging capacity, the essential oil samples at different concentration ranges (1-100 $\mu \mathrm{g} \mathrm{mL}^{-1}$ ) were mixed in the freshly DPPH solution $\left(0.1 \mathrm{mmol} \mathrm{L}^{-1}\right.$ in methanol, $\left.3.0 \mathrm{~mL}\right)$, according to previously described. ${ }^{21}$ The mixture was shaken vigorously and left to stand at room temperature in the dark. After $30 \mathrm{~min}$, absorbance $(517 \mathrm{~nm})$ was measured against a blank containing all reagents except the test samples, using an ultraviolet-visible (UV-Vis) spectrophotometer (Femto, model 700 plus). BHT was used as positive control.

The scavenging activity was calculated as equation 1 :

$\% \mathrm{DPPH}$ scavenging activity $=\left(\frac{\mathrm{A} 0-\mathrm{A}}{\mathrm{A} 0}\right) \times 100$

where: A0 is the absorbance of the blank solution and $\mathrm{A}$ is the absorbance of the essential oil. The percentage of scavenging activity was plotted against the sample concentration to obtain effective concentration $\left(\mathrm{IC}_{50}\right)$ defined as the concentration of the sample necessary to scavenge $50 \%$ of the DPPH radicals. Determinations were performed in triplicate.

\section{$\beta$-Carotene bleaching (BCB) assay}

The antioxidant activity of the essential oils were measured on the basis of the $\beta$-carotene bleaching system according to the previously reported. ${ }^{22}$ Briefly, $\beta$-carotene was dissolved in chloroform $\left(0.2 \mathrm{mg} \mathrm{mL}^{-1}\right)$ and an aliquot $(1 \mathrm{~mL})$ of this solution was mixed with linoleic acid (20 mg) and Tween-40 ${ }^{\circledR}$ (200 mg). Subsequently, chloroform was removed under vacuum using a rotary evaporator (Fisatom, model 801) and then distilled water saturated with oxygen $(50 \mathrm{~mL})$ was slowly added with vigorous agitation to form an emulsion. Emulsion aliquots $(5 \mathrm{~mL})$ were mixed with each essential oil sample $(0.2 \mathrm{~mL})$. Control samples were prepared with methanol $(0.2 \mathrm{~mL})$ without sample. BHT was used for comparative propose. As soon as the emulsion was added to each tube, the absorbance $(470 \mathrm{~nm})$ was measured (zero time) using a UV-Vis spectrophotometer (Femto, model 700 plus $)$. Tubes were placed in a water bath $\left(50^{\circ} \mathrm{C}\right)$ and oxidation was monitored by absorbance measurements at 15 min intervals until the color of $\beta$-carotene had disappeared in the control sample (approximately $105 \mathrm{~min}$ ). The experiments were carried out in triplicate.

Antioxidant activity (AA) was calculated as percent inhibition relative to the control (equation 2):

$\mathrm{AA}=100 \times\left(\frac{1-(\mathrm{Ai}-\mathrm{At})}{\left(\mathrm{A}^{\prime} \mathrm{i}-\mathrm{A}^{\prime} \mathrm{t}\right)}\right)$

where Ai: absorbance values $(0 \mathrm{~min})$ for the tested sample, At: absorbance values $(0 \mathrm{~min})$ for the control, A'i: absorbance value $\left(105 \mathrm{~min}, 50{ }^{\circ} \mathrm{C}\right)$ for the tested sample, and A't: absorbance value (105 min, $\left.50{ }^{\circ} \mathrm{C}\right)$ for the control. The experiments were performed in triplicate.

\section{Antifungal activity}

In order to evaluate the minimum inhibitory concentration (MIC) of the essential oils against the yeast Candida albicans (ATCC10231), a broth microdilution assay was performed according to the method previously described. ${ }^{23}$ Briefly, serial dilutions of each essential oil (2.0-50 $\left.\mu \mathrm{g} \mathrm{mL}^{-1}\right)$ and a volume of Sabouraud broth, inoculated with the microorganism $\left(10^{5}\right.$ colony-forming units (CFU) $\mathrm{mL}^{-1}$ ), were added in 96-well plates. The plates were incubated for $72 \mathrm{~h}$ at $25^{\circ} \mathrm{C}$. Experiments were performed in triplicate, nystatin was used as positive control and proper blanks were carried out simultaneously.

\section{Results and Discussion}

\section{Chemical constituents}

There was a variation in the yield of the essential oil according to the harvest time. The yield from spring, summer, and autumn leaves was $0.89,0.81$ and $0.70 \%$ respectively, while winter had the poorest yield by far, at only $0.05 \%$. This is probably due to Dourado's climate at that time of year; while summers tend to be extremely hot 
and sunny, on winter the weather is milder, with an extreme low amount of rainfall. As a consequence, the essential oil from winter was only used for chemical characterization; the amount obtained was not enough for antioxidant and antifungal assays.

In the beginning of the chemical composition study, the performance of the rapid-scanning qMS for identification of essential oil compounds with a high level of confidence was evaluated. To assess the performance, a set of essential oil standard compounds of different classes-namely monoterpenes hydrocarbons ( $\beta$-pinene and limonene), oxygenated monoterpenes (1,8-cineole, linalool, camphor, $\alpha$-terpineol, and carvone), sesquiterpenes hydrocarbons ((E)-caryophyllene and $\alpha$-humulene), and oxygenated sesquiterpenes $((E)$-nerolidol and $\alpha$-bisabolol)-were used to evaluated important qMS parameters (number of data points per peak, the average spectra quality match, and the peak skewing). Therefore, the mass spectrometer was operated in fast scan mode (20000 amu s $\mathrm{s}^{-1}$ ) over a $40-250 \mathrm{~m} / \mathrm{z}$ range, giving a $65 \mathrm{~Hz}$ acquisition rate.

Table 1 reports the number of modulations per peak, data points, peak weight, average spectra quality match (MS\%) and the selected ions (target ion (T), and qualifier ions (Q1 and Q2)) used to evaluate the skewing of the mass spectra, for each investigated compound.

The system employed generated at least 7 data points per peak, for all analyzed compounds. These results are considered sufficient amount of data points. ${ }^{24}$ Even for the group of compounds that showed the lowest number of data points ( $\beta$-pinene, $\alpha$-terpineol and $(E)$-nerolidol), the results were satisfactory. The majority of evaluated compounds showed peak width of $180 \mathrm{~ms}$ with number of scans per peak ranging from 9 to 11 .
The quality of the mass spectra was measured by the similarity match (\%MS). It was obtained by the comparison of experimental mass spectra and mass spectra from commercial library (NIST), and was evaluated at each scan of the main modulated peak, for each investigated compound. According to the results, good similarity match was obtained with an average from 92 to $94 \%$. Furthermore, the low percent relative standard deviation (\%RSD) values for this parameter showed that $\% \mathrm{MS}$ were constant across the evaluated peaks.

The compounds showed some mass spectral distortion across their chromatographic peak. As can be seen in Table 1 , the $\%$ RSD values for the ratios $T / Q_{1}$ and $T / Q_{2}$ ranged from 4.8 to $9.8 \%$. It means that mass ratios (or mass spectral) were not even across the peak elution. However, these \% RSD values are considered synonymous of relative stability for this parameter. ${ }^{16}$

The high qMS performance observed with the standard compounds (high similarity match and acceptable stability of the mass ratio) allowed us to adopt this experimental setting to characterize complex samples as P. glabratum essential oil with high level of confidence. Figure 1 illustrates a total ion chromatogram from the $\mathrm{GC} \times \mathrm{GC} / \mathrm{qMS}$ presented as a contour plot for the essential oil samples.

Considering the four essential oils, a total of 199 compounds were identified, with satisfactory library matches and LTPRIs. The experimental LTPRIs showed a good concordance with those reported by Adams ${ }^{20}$ for 1D-GC. The identified compounds are listed in Table 2 with their LTPRI from the literature, LTPRI differences (experimental LTPRI - literature LTPRI), and relative percentage of the chromatographic area.

Table 1. Number of modulations per peak; number of points per peak for the main modulated peak; peak width at the baseline; average spectral similarity (\%MS); target ion $(\mathrm{T})$, and qualifier ions $\left(\mathrm{Q}_{1}\right.$ and $\left.\mathrm{Q}_{2}\right)$ ratios, with their percent relative standard deviation (\%RSD) for each 2D peak

\begin{tabular}{|c|c|c|c|c|c|c|c|}
\hline No. & Compound & $\begin{array}{l}\text { Modulations per } \\
\text { peak }\end{array}$ & $\begin{array}{c}\text { Number of } \\
\text { points in main } \\
\text { modulation peak }\end{array}$ & $\begin{array}{l}\text { Peak width at } \\
\text { baseline / ms }\end{array}$ & MS (RSD) / \% & $\mathrm{T} / \mathrm{Q}_{1}(\mathrm{RSD}) / \%$ & $\mathrm{~T} / \mathrm{Q}_{2}(\mathrm{RSD}) / \%$ \\
\hline 1 & $\beta$-pinene & 4 & 7 & 120 & $92.0(1.9)$ & $93 / 69(9.4)$ & $93 / 79(9.1)$ \\
\hline 2 & limonene & 3 & 8 & 120 & $92.6(1.1)$ & $68 / 93(8.4)$ & $68 / 67(8.2)$ \\
\hline 3 & 1,8-cineole & 3 & 10 & 180 & $92.2(1.6)$ & $81 / 93(9.8)$ & $81 / 108(8.1)$ \\
\hline 4 & linalool & 5 & 10 & 180 & $92.7(2.0)$ & $71 / 93(9.3)$ & $71 / 55(8.1)$ \\
\hline 5 & camphor & 5 & 11 & 180 & $93.0(3.0)$ & $95 / 81(4.8)$ & 95/108 (8.9) \\
\hline 6 & $\alpha$-terpineol & 3 & 7 & 120 & $93.5(1.8)$ & $59 / 93(9.8)$ & $59 / 121(9.1)$ \\
\hline 7 & carvone & 5 & 9 & 180 & $92.4(2.0)$ & $82 / 54(8.9)$ & 82/108 (8.8) \\
\hline 8 & (E)-caryophyllene & 4 & 9 & 180 & $93.0(1.2)$ & $93 / 69(7.9)$ & 93/91 (5.6) \\
\hline 9 & $\alpha$-humulene & 5 & 9 & 180 & $94.0(0.7)$ & $93 / 80(8.8)$ & 93/121 (7.8) \\
\hline 10 & (E)-nerolidol & 5 & 7 & 120 & $93.0(1.1)$ & $69 / 93(5.5)$ & $69 / 107(9.3)$ \\
\hline 11 & $\alpha$-bisabolol & 5 & 10 & 180 & $92.2(1.9)$ & $69 / 109(9.5)$ & $69 / 119(9.7)$ \\
\hline
\end{tabular}



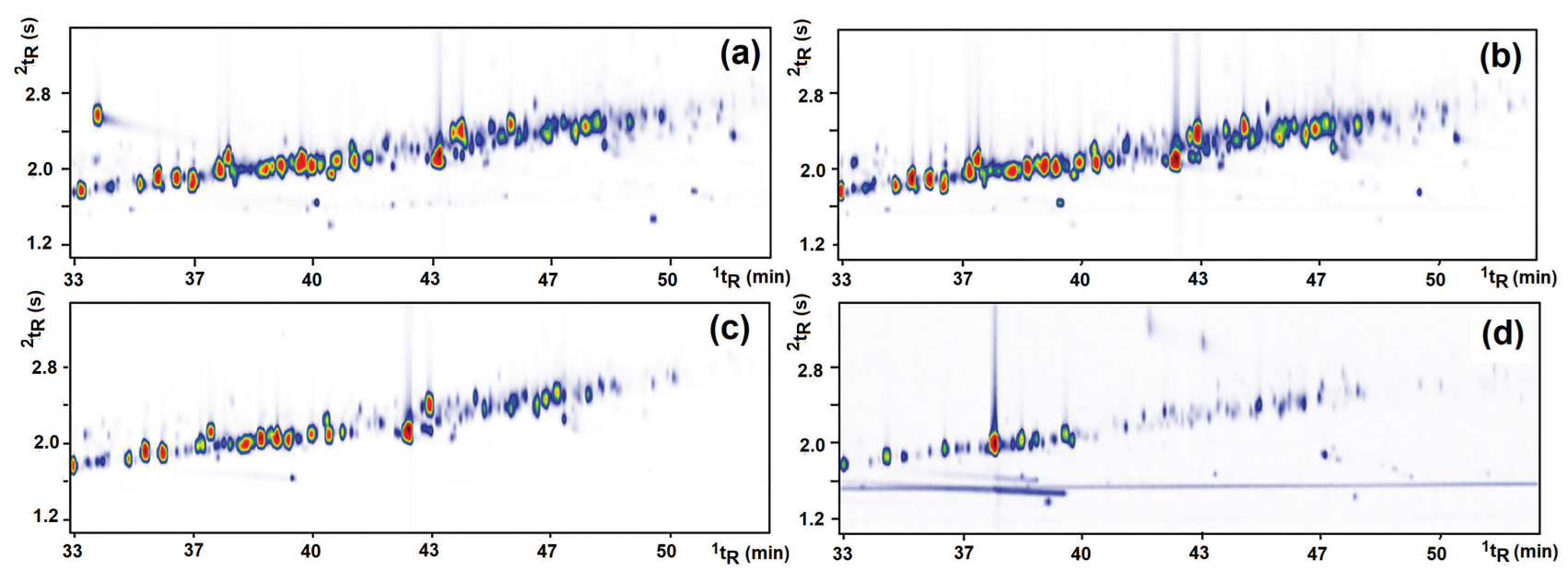

Figure 1. Total ion chromatogram presented as contour plot from essential oils of summer (a), spring (b), autumn (c), and winter (d) leaves of P. glabratum.

Table 2. The list of identified compounds, literature LTPRI values, LTPRI differences and their percentage composition in the essential oils from Piper glabratum analyzed by GC×GC/qMS

\begin{tabular}{|c|c|c|c|c|c|c|c|c|c|c|}
\hline \multirow{2}{*}{ No. } & \multirow{2}{*}{ Compound } & \multirow{2}{*}{ LTPRI $_{\mathrm{lit}}{ }^{\mathrm{a}}$} & \multicolumn{2}{|c|}{ Spring } & \multicolumn{2}{|c|}{ Summer } & \multicolumn{2}{|c|}{ Autumn } & \multicolumn{2}{|c|}{ Winter } \\
\hline & & & $\Delta_{\text {LTPRI }}^{\text {b }}$ & $\mathrm{V}^{\mathrm{c}} / \%$ & $\Delta_{\text {LTPRI }}^{\mathrm{b}}$ & $\mathrm{V}^{\mathrm{c}} / \%$ & $\Delta_{\text {LTPRI }}{ }^{\mathrm{b}}$ & $\mathrm{V}^{\mathrm{c}} / \%$ & $\Delta_{\text {LTPRI }}$ b & $\mathrm{V}^{\mathrm{c}} / \%$ \\
\hline 1 & isocitronellene & 918 & 5 & 0.01 & n.i. & n.i. & n.i. & n.i. & n.i. & n.i. \\
\hline 2 & tricyclene & 921 & 6 & 0.05 & 2 & 0.13 & 2 & 0.31 & n.i. & n.i. \\
\hline 3 & $\operatorname{artemisia~triene~}^{\mathrm{d}}$ & 923 & 5 & 0.01 & 7 & 8.47 & 7 & 6.40 & n.i. & n.i. \\
\hline 4 & camphene & 946 & n.i. & n.i. & -4 & 0.07 & -4 & 0.13 & n.i. & n.i. \\
\hline 5 & $m$-ethyltoluene & 958 & -1 & 0.02 & n.i. & n.i. & n.i. & n.i. & n.i. & n.i. \\
\hline 6 & $p$-ethyltoluene & 960 & -2 & 0.02 & n.i. & n.i. & n.i. & n.i. & n.i. & n.i. \\
\hline 7 & heptan-2-ol 6-methyl & 958 & n.i. & n.i. & 5 & 0.01 & n.i. & n.i. & n.i. & n.i. \\
\hline 8 & sabinene & 969 & 0 & 0.14 & n.i. & n.i. & n.i. & n.i. & n.i. & n.i. \\
\hline 9 & $\beta$-pinene & 974 & -4 & 0.01 & -2 & 9.66 & -4 & 6.75 & n.i. & n.i. \\
\hline 10 & 1-octen-3-ol & 974 & 8 & 0.02 & n.i. & n.i. & n.i. & n.i. & n.i. & n.i. \\
\hline 11 & 6-methyl-5-hepten-2-one & 981 & n.i. & n.i. & 7 & 0.97 & 4 & 0.78 & n.i. & n.i. \\
\hline 12 & $\Psi$-cumene $e^{\mathrm{d}, e}$ & 988 & 0 & 0.03 & n.i. & n.i. & n.i. & n.i. & n.i. & n.i. \\
\hline 13 & myrcene $^{d}$ & 988 & 2 & 0.02 & 2 & 1.26 & 0 & 0.88 & n.i. & n.i. \\
\hline 14 & 3-octanol & 988 & 10 & 0.02 & n.i. & n.i. & n.i. & n.i. & n.i. & n.i. \\
\hline 15 & decane & 1000 & n.i. & n.i. & 0 & 0.05 & n.i. & n.i. & n.i. & n.i. \\
\hline 16 & $\alpha$-phellandrene ${ }^{\mathrm{d}}$ & 1002 & 3 & 0.02 & -2 & 0.02 & -4 & 0.07 & n.i. & n.i. \\
\hline 17 & $\alpha$-terpinene $\mathrm{d}^{\mathrm{d}}$ & 1014 & n.i. & n.i. & -1 & 0.29 & -4 & 0.19 & n.i. & n.i. \\
\hline 18 & $p$-cymene $e^{\mathrm{d}, e}$ & 1020 & n.i. & n.i. & 2 & 0.11 & -2 & 0.07 & n.i. & n.i. \\
\hline 19 & limonene & 1024 & 1 & 1.14 & 1 & 2.80 & -2 & 2.52 & n.i. & n.i. \\
\hline 20 & 1,8-cineole & 1026 & 0 & 0.85 & n.i. & n.i. & n.i. & n.i. & n.i. & n.i. \\
\hline 21 & indane & 1035 & n.i. & n.i. & -5 & 0.01 & n.i. & n.i. & n.i. & n.i. \\
\hline 22 & $(Z)-\beta$-ocimene ${ }^{d}$ & 1032 & 6 & 0.06 & 6 & 0.62 & 3 & 1.08 & n.i. & n.i. \\
\hline 23 & $(\boldsymbol{E})$ - $\boldsymbol{\beta}$-ocimene $\mathrm{d}^{\mathrm{d}}$ & 1044 & 4 & 0.15 & 4 & 0.21 & 1 & 0.34 & n.i. & n.i. \\
\hline 24 & $\gamma$-terpinene & 1054 & 3 & 0.02 & 3 & 0.68 & -1 & 0.49 & n.i. & n.i. \\
\hline 25 & cis-sabinene hydrate & 1065 & 0 & 0.08 & 2 & 0.05 & -3 & 0.06 & n.i. & n.i. \\
\hline 26 & 2-ethyl-p-xylene ${ }^{\mathrm{d}, \mathrm{e}}$ & 1085 & -10 & 0.02 & -10 & 0.02 & n.i. & n.i. & n.i. & n.i. \\
\hline 27 & 4-ethyl-m-xylene ${ }^{\mathrm{d}, \mathrm{e}}$ & 1086 & -4 & 0.01 & -4 & 0.01 & n.i. & n.i. & n.i. & n.i. \\
\hline 28 & terpinolene & 1086 & -1 & 0.02 & -1 & 0.28 & -4 & 0.19 & n.i. & n.i. \\
\hline 29 & 4-ethyl-o-xylene ${ }^{\mathrm{d}, \mathrm{e}}$ & 1092 & -4 & 0.01 & n.i. & n.i. & n.i. & n.i. & n.i. & n.i. \\
\hline 30 & trans-sabinene hydrate & 1098 & n.i. & n.i. & -1 & 0.04 & -5 & 0.05 & n.i. & n.i. \\
\hline 31 & linalool & 1095 & 7 & 0.10 & 5 & 0.07 & 2 & 0.05 & n.i. & n.i. \\
\hline 32 & exo-fenchol & 1118 & n.i. & n.i. & -8 & 0.04 & n.i. & n.i. & n.i. & n.i. \\
\hline 33 & perillene $^{\mathrm{d}}$ & 1102 & n.i. & n.i. & 15 & 0.40 & 12 & 0.76 & n.i. & n.i. \\
\hline
\end{tabular}


Table 2. The list of identified compounds, literature LTPRI values, LTPRI differences and their percentage composition in the essential oils from Piper glabratum analyzed by GC×GC/qMS (cont.)

\begin{tabular}{|c|c|c|c|c|c|c|c|c|c|c|}
\hline \multirow{2}{*}{ No. } & \multirow{2}{*}{ Compound } & \multirow{2}{*}{ LTPRI $_{\text {lit }}{ }^{a}$} & \multicolumn{2}{|c|}{ Spring } & \multicolumn{2}{|c|}{ Summer } & \multicolumn{2}{|c|}{ Autumn } & \multicolumn{2}{|c|}{ Winter } \\
\hline & & & $\Delta_{\text {LTPRI }}{ }^{\mathrm{b}}$ & $\mathrm{V}^{\mathrm{c}} / \%$ & $\Delta_{\text {LTPRI }}{ }^{\mathrm{b}}$ & $\mathrm{V}^{\mathrm{c}} / \%$ & $\Delta_{\text {LTPRI }}{ }^{\mathrm{b}}$ & $\mathrm{V}^{\mathrm{c}} / \%$ & $\Delta_{\text {LTPRI }}{ }^{\mathrm{b}}$ & $\mathrm{V}^{\mathrm{c}} / \%$ \\
\hline 34 & $1,3,8-p$-menthatriene ${ }^{d}$ & 1108 & 4 & 0.01 & n.i. & n.i. & 8 & 0.05 & n.i. & n.i. \\
\hline 35 & isodurene $^{\mathrm{d}, \mathrm{e}}$ & 1129 & -12 & 0.01 & n.i. & n.i. & n.i. & n.i. & n.i. & n.i. \\
\hline 36 & $c i s-p$-menth-2-en-1-ol & 1118 & 3 & 0.02 & 3 & 0.06 & n.i. & n.i. & n.i. & n.i. \\
\hline 37 & methyl octanoate & 1123 & 5 & 0.02 & n.i. & n.i. & n.i. & n.i. & n.i. & n.i. \\
\hline 38 & $(E)$-limonene oxide & 1132 & n.i. & n.i. & 4 & 0.03 & n.i. & n.i. & n.i. & n.i. \\
\hline 39 & trans-p-menth-2-em-1-ol & 1136 & 4 & 0.01 & 4 & 0.03 & 0 & 0.02 & n.i. & n.i. \\
\hline 40 & (Z)-myroxide ${ }^{\mathrm{d}}$ & 1131 & n.i. & n.i. & n.i. & n.i. & 9 & 0.02 & n.i. & n.i. \\
\hline 41 & $(E)$-myroxide ${ }^{\mathrm{d}}$ & 1140 & 5 & 0.04 & n.i. & n.i. & 2 & 0.03 & n.i. & n.i. \\
\hline 42 & camphor & 1141 & n.i. & n.i. & 1 & 0.03 & n.i. & n.i. & n.i. & n.i. \\
\hline 43 & camphene hydrate & 1145 & n.i. & n.i. & 0 & 0.05 & n.i. & n.i. & n.i. & n.i. \\
\hline 44 & prehnitol $^{\mathrm{d}, e}$ & 1148 & 0 & 0.02 & 0 & 0.01 & n.i. & n.i. & n.i. & n.i. \\
\hline 45 & pinocarvone & 1160 & n.i. & n.i. & 1 & 0.13 & -3 & 0.06 & n.i. & n.i. \\
\hline 46 & isoborneol & 1155 & n.i. & n.i. & 9 & 0.03 & n.i. & n.i. & n.i. & n.i. \\
\hline 47 & $\delta$-terpineol & 1162 & 5 & 0.08 & n.i. & n.i. & n.i. & n.i. & n.i. & n.i. \\
\hline 48 & (2E)-nonen-1-al & 1157 & n.i. & n.i. & 12 & 0.02 & n.i. & n.i. & n.i. & n.i. \\
\hline 49 & cis-pinocamphone & 1172 & 1 & 0.00 & 1 & 0.05 & n.i. & n.i. & n.i. & n.i. \\
\hline 50 & thuj-3-en-10-al & 1181 & n.i. & n.i. & -5 & 0.03 & n.i. & n.i. & n.i. & n.i. \\
\hline 51 & terpinen-4-ol & 1174 & 2 & 0.23 & 2 & 0.77 & -1 & 0.65 & n.i. & n.i. \\
\hline 52 & naphthalene $e^{\mathrm{d}, \mathrm{e}}$ & 1178 & 0 & 0.01 & n.i. & n.i. & n.i. & n.i. & n.i. & n.i. \\
\hline 53 & myrtanal & 1198 & n.i. & n.i. & -19 & 0.01 & n.i. & n.i. & n.i. & n.i. \\
\hline 54 & $\alpha$-terpineol & 1186 & 6 & 0.43 & 4 & 0.43 & 0 & 0.29 & n.i. & n.i. \\
\hline 55 & myrtenol & 1219 & n.i. & n.i. & -20 & 0.04 & -20 & 0.03 & n.i. & n.i. \\
\hline 56 & dodecane & 1200 & n.i. & n.i. & 2 & 0.02 & n.i. & n.i. & n.i. & n.i. \\
\hline 57 & trans-piperitol & 1207 & 2 & 0.01 & n.i. & n.i. & n.i. & n.i. & n.i. & n.i. \\
\hline 58 & (E)-2-dodecene & 1205 & 4 & 0.01 & n.i. & n.i. & n.i. & n.i. & n.i. & n.i. \\
\hline 59 & verbenone & 1204 & n.i. & n.i. & 5 & 0.01 & n.i. & n.i. & n.i. & n.i. \\
\hline 60 & trans-carveol & 1215 & 5 & 0.01 & 5 & 0.02 & n.i. & n.i. & n.i. & n.i. \\
\hline 61 & carvone & 1243 & 3 & 0.04 & n.i. & n.i. & n.i. & n.i. & n.i. & n.i. \\
\hline 62 & cis-myrtanol & 1250 & 5 & 0.01 & 3 & 0.01 & n.i. & n.i. & n.i. & n.i. \\
\hline 63 & piperitone & 1249 & n.i. & n.i. & 6 & 0.01 & n.i. & n.i. & n.i. & n.i. \\
\hline 64 & (4Z)-decen-1-ol & 1255 & n.i. & n.i. & 1 & 0.02 & n.i. & n.i. & n.i. & n.i. \\
\hline 65 & trans-myrtanol & 1258 & n.i. & n.i. & 2 & 0.02 & n.i. & n.i. & n.i. & n.i. \\
\hline 66 & $(4 E)$-decen-1-ol & 1259 & n.i. & n.i. & 3 & 0.02 & n.i. & n.i. & n.i. & n.i. \\
\hline 67 & $p$-propylphenol ${ }^{\mathrm{d}, \mathrm{e}}$ & 1260 & 6 & 0.05 & n.i. & n.i. & n.i. & n.i. & n.i. & n.i. \\
\hline 68 & $o$-tert-butylphenol ${ }^{\mathrm{d}, \mathrm{e}}$ & 1274 & 6 & 0.01 & n.i. & n.i. & n.i. & n.i. & n.i. & n.i. \\
\hline 69 & $p$-sec-butylphenol ${ }^{\mathrm{d}, e}$ & 1279 & 7 & 0.02 & n.i. & n.i. & n.i. & n.i. & n.i. & n.i. \\
\hline 70 & isobornyl acetate & 1285 & 2 & 0.02 & 1 & 0.06 & -1 & 0.05 & n.i. & n.i. \\
\hline 71 & $(\boldsymbol{E})$-anethole ${ }^{\mathrm{d}, \mathrm{e}}$ & 1282 & 6 & 0.01 & n.i. & n.i. & n.i. & n.i. & n.i. & n.i. \\
\hline 72 & lavandulyl acetate & 1288 & n.i. & n.i. & n.i. & n.i. & 7 & 0.05 & n.i. & n.i. \\
\hline 73 & 3-thujyl acetate & 1290 & n.i. & n.i. & 6 & 0.06 & n.i. & n.i. & n.i. & n.i. \\
\hline 74 & trans-pinocarvyl acetate & 1298 & 4 & 0.02 & 0 & 0.03 & -1 & 0.04 & n.i. & n.i. \\
\hline 75 & $(2 E, 4 Z)$-decadienal & 1292 & n.i. & n.i. & n.i. & n.i. & 6 & 0.01 & n.i. & n.i. \\
\hline 76 & tridecane & 1300 & n.i. & n.i. & 2 & 0.02 & n.i. & n.i. & n.i. & n.i. \\
\hline 77 & $(Z)$-methyl cinnamate ${ }^{\mathrm{d}, \mathrm{e}}$ & 1299 & 9 & 1.14 & n.i. & n.i. & n.i. & n.i. & n.i. & n.i. \\
\hline 78 & cis-pinocarvyl acetate & 1311 & 4 & 0.05 & 0 & 0.13 & -2 & 0.12 & n.i. & n.i. \\
\hline 79 & myrtenyl acetate & 1324 & n.i. & n.i. & 0 & 0.35 & -2 & 0.14 & n.i. & n.i. \\
\hline 80 & methyl decanoate & 1323 & 4 & 0.72 & n.i. & n.i. & n.i. & n.i. & n.i. & n.i. \\
\hline 81 & methyl geranate & 1322 & 5 & 0.16 & n.i. & n.i. & n.i. & n.i. & n.i. & n.i. \\
\hline 82 & $(Z)$-isosafrole ${ }^{\mathrm{d}, \mathrm{e}}$ & 1336 & -8 & 0.02 & n.i. & n.i. & n.i. & n.i. & n.i. & n.i. \\
\hline 83 & $\alpha$-terpinyl acetate & 1346 & n.i. & n.i. & -11 & 0.27 & -13 & 0.17 & n.i. & n.i. \\
\hline
\end{tabular}


Table 2. The list of identified compounds, literature LTPRI values, LTPRI differences and their percentage composition in the essential oils from Piper glabratum analyzed by GC×GC/qMS (cont.)

\begin{tabular}{|c|c|c|c|c|c|c|c|c|c|c|}
\hline \multirow{2}{*}{ No. } & \multirow{2}{*}{ Compound } & \multirow{2}{*}{ LTPRI $_{\text {lit }}{ }^{a}$} & \multicolumn{2}{|c|}{ Spring } & \multicolumn{2}{|c|}{ Summer } & \multicolumn{2}{|c|}{ Autumn } & \multicolumn{2}{|c|}{ Winter } \\
\hline & & & $\Delta_{\text {LTPRI }}{ }^{\mathrm{b}}$ & $\mathrm{V}^{\mathrm{c}} / \%$ & $\Delta_{\text {LTPRI }}^{\mathrm{b}}$ & $\mathrm{V}^{\mathrm{c}} / \%$ & $\Delta_{\text {LTPRI }}{ }^{\mathrm{b}}$ & $\mathrm{V}^{\mathrm{c}} / \%$ & $\Delta_{\text {LTPRI }}{ }^{\mathrm{b}}$ & $\mathrm{V}^{\mathrm{c}} / \%$ \\
\hline 84 & 7-epi-silphiperfol-5-ene & 1345 & n.i. & n.i. & -9 & 0.03 & -10 & 0.03 & n.i. & n.i. \\
\hline 85 & $\delta$-elemene & 1335 & 1 & 0.13 & n.i. & n.i. & n.i. & n.i. & -2 & 4.53 \\
\hline 86 & $\alpha$-cubebene & 1345 & 4 & 0.54 & 2 & 0.76 & 1 & 0.75 & n.i. & n.i. \\
\hline 87 & trans-carvyl acetate & 1339 & 12 & 0.03 & 10 & 0.06 & 8 & 0.06 & n.i. & n.i. \\
\hline 88 & cyclosativene & 1369 & -7 & 0.90 & -9 & 1.08 & -11 & 0.93 & n.i. & n.i. \\
\hline 89 & neoiso-dihydro carveol acetate & 1356 & 8 & 0.01 & n.i. & n.i. & n.i. & n.i. & n.i. & n.i. \\
\hline 90 & cis-carvyl acetate & 1365 & 1 & 0.03 & -3 & 0.05 & -5 & 0.05 & n.i. & n.i. \\
\hline 91 & $\alpha$-ylangene & 1373 & -2 & 0.12 & -6 & 0.14 & -7 & 0.21 & n.i. & n.i. \\
\hline 92 & linalool isobutanoate & 1373 & -2 & 0.02 & -6 & 0.04 & -7 & 0.02 & n.i. & n.i. \\
\hline 93 & $\alpha$-copaene & 1374 & 1 & 2.81 & -1 & 2.59 & -3 & 2.21 & -5 & 0.53 \\
\hline 94 & isoledene & 1374 & 6 & 0.02 & 2 & 0.02 & n.i. & n.i. & n.i. & n.i. \\
\hline 95 & $\beta$-bourbonene & 1387 & -3 & 0.11 & -7 & 0.10 & -7 & 0.25 & -9 & 1.04 \\
\hline 96 & trans-myrtanol acetate & 1385 & -1 & 0.10 & -5 & 0.17 & n.i. & n.i. & n.i. & n.i. \\
\hline 97 & $(E)$-methyl cinnamate ${ }^{\mathrm{d}, \mathrm{e}}$ & 1376 & 10 & 3.31 & n.i. & n.i. & n.i. & n.i. & n.i. & n.i. \\
\hline 98 & $\beta$-cubebene & 1387 & n.i. & n.i. & 0 & 0.29 & -1 & 0.15 & n.i. & n.i. \\
\hline 99 & $\alpha$-duprezianene & 1387 & 4 & 0.01 & n.i. & n.i. & n.i. & n.i. & n.i. & n.i. \\
\hline 100 & $\beta$-elemene & 1389 & 4 & 0.53 & n.i. & n.i. & 0 & 0.46 & -2 & 1.19 \\
\hline 101 & $(2 E)$-decenyl acetate & 1408 & -12 & 0.05 & -15 & 0.10 & n.i. & n.i. & n.i. & n.i. \\
\hline 102 & 7-epi-sesquithujene & 1390 & 7 & 0.02 & 5 & 0.02 & 3 & 0.04 & n.i. & n.i. \\
\hline 103 & tetradecane & 1400 & n.i. & n.i. & 0 & 0.02 & n.i. & n.i. & n.i. & n.i. \\
\hline 104 & $\alpha$-gurjunene & 1409 & 0 & 1.48 & -4 & 1.40 & 0 & 1.46 & -5 & 0.10 \\
\hline 105 & $\alpha$-cedrene & 1410 & -1 & 0.01 & n.i. & n.i. & n.i. & n.i. & n.i. & n.i. \\
\hline 106 & (E)-caryophyllene & 1417 & 2 & 4.89 & -1 & 4.16 & -3 & 4.90 & -3 & 2.48 \\
\hline 107 & $\beta$-copaene & 1430 & -1 & 3.51 & -4 & 3.35 & -6 & 3.98 & -7 & 0.38 \\
\hline 108 & $\gamma$-elemene & 1434 & -5 & 0.04 & -8 & 0.04 & n.i. & n.i. & n.i. & n.i. \\
\hline 109 & $\alpha$-trans-bergamotene & 1432 & 4 & 4.31 & 1 & 2.04 & n.i. & n.i. & n.i. & n.i. \\
\hline 110 & neryl acetone & 1434 & n.i. & n.i. & 1 & 0.04 & n.i. & n.i. & n.i. & n.i. \\
\hline 111 & aromadendrene & 1439 & n.i. & n.i. & n.i. & n.i. & -6 & 0.13 & -1 & 0.15 \\
\hline 112 & $\alpha$-guaiene & 1437 & n.i. & n.i. & 3 & 0.12 & 1 & 0.12 & n.i. & n.i. \\
\hline 113 & cis-muurola-3,5-diene $\mathrm{d}^{\mathrm{d}}$ & 1448 & n.i. & n.i. & n.i. & n.i. & -7 & 0.07 & n.i. & n.i. \\
\hline 114 & trans-muurola-3,5-diene $^{d}$ & 1451 & n.i. & n.i. & n.i. & n.i. & -6 & 0.61 & n.i. & n.i. \\
\hline 115 & $\alpha$-humulene & 1452 & 0 & 4.41 & -4 & 3.13 & -4 & 1.65 & -5 & 1.27 \\
\hline 116 & geranyl acetone & 1453 & 4 & 3.15 & 1 & 2.89 & -1 & 2.74 & n.i. & n.i. \\
\hline 117 & $\beta$-santalene & 1457 & n.i. & n.i. & 0 & 0.33 & n.i. & n.i. & n.i. & n.i. \\
\hline 118 & allo-aromadendrene & 1458 & 1 & 1.11 & n.i. & n.i. & n.i. & n.i. & -4 & 0.27 \\
\hline 119 & $(E)-\beta$-farnesene $e^{d}$ & 1454 & n.i. & n.i. & n.i. & n.i. & 3 & 0.07 & n.i. & n.i. \\
\hline 120 & 9-epi-(E)-caryophyllene & 1464 & 2 & 0.01 & n.i. & n.i. & n.i. & n.i. & -4 & 0.18 \\
\hline 121 & drima-7,9(11)-diene ${ }^{\mathrm{d}}$ & 1469 & 0 & 0.29 & -3 & 0.31 & -5 & 0.70 & n.i. & n.i. \\
\hline 122 & trans-cadina-1(6),4-diene $\mathrm{d}^{\mathrm{d}}$ & 1475 & 3 & 3.30 & n.i. & n.i. & n.i. & n.i. & n.i. & n.i. \\
\hline 123 & germacrene $\mathbf{D}^{\mathrm{d}}$ & 1484 & -3 & 1.03 & -10 & 3.38 & -11 & 5.47 & -8 & 77.74 \\
\hline 124 & $\beta$-selinene & 1489 & -3 & 3.46 & -8 & 3.31 & -8 & 4.84 & n.i. & n.i. \\
\hline 125 & $(E)-\beta$-ionone ${ }^{d}$ & 1487 & 1 & 0.08 & -2 & 0.04 & n.i. & n.i. & n.i. & n.i. \\
\hline 126 & trans-muurola-4(14),5-diene $\mathrm{d}^{\mathrm{d}}$ & 1493 & -1 & 0.62 & n.i. & n.i. & n.i. & n.i. & -7 & 0.39 \\
\hline 127 & $\alpha$-selinene & 1498 & n.i. & n.i. & -6 & 4.29 & -6 & 6.19 & n.i. & n.i. \\
\hline 128 & bicyclogermacrene & 1500 & -3 & 6.93 & n.i. & n.i. & n.i. & n.i. & -8 & 3.49 \\
\hline 129 & $\alpha$-muurolene & 1500 & 2 & 3.88 & -2 & 3.08 & -2 & 3.50 & -3 & 0.18 \\
\hline 130 & pentadecane & 1500 & n.i. & n.i. & 0 & 0.19 & 0 & 0.12 & n.i. & n.i. \\
\hline 131 & $\delta$-amorphene & 1511 & n.i. & n.i. & -6 & 0.26 & -8 & 0.45 & n.i. & n.i. \\
\hline 132 & trans-cycloisolongifol-5-ol & 1513 & -8 & 0.04 & n.i. & n.i. & n.i. & n.i. & n.i. & n.i. \\
\hline 133 & $\alpha$-bulnesene & 1509 & -2 & 1.11 & n.i. & n.i. & n.i. & n.i. & n.i. & n.i. \\
\hline
\end{tabular}


Table 2. The list of identified compounds, literature LTPRI values, LTPRI differences and their percentage composition in the essential oils from Piper glabratum analyzed by GC×GC/qMS (cont.)

\begin{tabular}{|c|c|c|c|c|c|c|c|c|c|c|}
\hline \multirow{2}{*}{ No. } & \multirow{2}{*}{ Compound } & \multirow{2}{*}{ LTPRI $_{\text {lit }}{ }^{a}$} & \multicolumn{2}{|c|}{ Spring } & \multicolumn{2}{|c|}{ Summer } & \multicolumn{2}{|c|}{ Autumn } & \multicolumn{2}{|c|}{ Winter } \\
\hline & & & $\Delta_{\text {LTPRI }}{ }^{\mathrm{b}}$ & $\mathrm{V}^{\mathrm{c}} / \%$ & $\Delta_{\text {LTPRI }}{ }^{\mathrm{b}}$ & $\mathrm{V}^{\mathrm{c}} / \%$ & $\Delta_{\text {LTPRI }}^{\mathrm{b}}$ & $\mathrm{V}^{\mathrm{c}} / \%$ & $\Delta_{\text {LTPRI }}{ }^{\mathrm{b}}$ & $\mathrm{V}^{\mathrm{c}} / \%$ \\
\hline 134 & $(E, E)-\alpha$-farnesene ${ }^{\mathrm{d}}$ & 1505 & 8 & 1.50 & 3 & 0.42 & n.i. & n.i. & n.i. & n.i. \\
\hline 135 & $\gamma$-cadinene & 1513 & 2 & 2.45 & -1 & 1.81 & -3 & 2.42 & -3 & 0.29 \\
\hline 136 & elixene & 1511 & n.i. & n.i. & n.i. & n.i. & n.i. & n.i. & 6 & 2.59 \\
\hline 137 & trans-calamenene $\mathrm{e}^{\mathrm{d}, \mathrm{e}}$ & 1521 & 3 & 0.45 & -1 & 0.52 & -1 & 0.99 & n.i. & n.i. \\
\hline 138 & $\delta$-cadinene & 1522 & 3 & 2.83 & 0 & 2.52 & -2 & 2.87 & -2 & 0.99 \\
\hline 139 & trans-cadina-1,4-diene & 1533 & 0 & 1.04 & -5 & 0.87 & -5 & 0.98 & n.i. & n.i. \\
\hline 140 & $\alpha$-cadinene & 1537 & 1 & 0.18 & -4 & 0.10 & -4 & 0.22 & n.i. & n.i. \\
\hline 141 & selina-3,7(11)-diene & 1545 & n.i. & n.i. & -8 & 0.02 & -8 & 0.04 & n.i. & n.i. \\
\hline 142 & $\boldsymbol{\alpha}$-calacorene $e^{\mathrm{d}, e}$ & 1544 & n.i. & n.i. & n.i. & n.i. & -5 & 0.15 & n.i. & n.i. \\
\hline 143 & 10-epi-cubebol & 1533 & 14 & 0.10 & 10 & 0.11 & 10 & 0.07 & n.i. & n.i. \\
\hline 144 & elemol & 1548 & n.i. & n.i. & 4 & 0.13 & -1 & 0.09 & n.i. & n.i. \\
\hline 145 & germacrene B & 1559 & -4 & 0.12 & n.i. & n.i. & -9 & 0.29 & -9 & 0.21 \\
\hline 146 & ledol & 1565 & 7 & 0.16 & 10 & 0.03 & n.i. & n.i. & n.i. & n.i. \\
\hline 147 & $\boldsymbol{\beta}$-calacorene $\mathrm{e}^{\mathrm{d}, \mathrm{e}}$ & 1564 & n.i. & n.i. & -5 & 0.03 & -5 & 0.04 & n.i. & n.i. \\
\hline 148 & germacrene D-4-ol & 1574 & -9 & 0.16 & n.i. & n.i. & n.i. & n.i. & n.i. & n.i. \\
\hline 149 & $(E)$-nerolidol & 1569 & 1 & 8.45 & -4 & 6.76 & -2 & 8.86 & n.i. & n.i. \\
\hline 150 & spathulenol & 1577 & 0 & 1.33 & -3 & 0.67 & -3 & 0.23 & -5 & 0.27 \\
\hline 151 & caryophyllene oxide & 1582 & 0 & 5.56 & -3 & 4.67 & -5 & 4.40 & -5 & 0.19 \\
\hline 152 & gleenol & 1586 & 1 & 0.38 & -3 & 0.37 & -4 & 0.40 & n.i. & n.i. \\
\hline 153 & viridiflorol & 1592 & -2 & 0.75 & -5 & 0.14 & -5 & 0.09 & n.i. & n.i. \\
\hline 154 & salvial-4(14)-en-1-one & 1594 & n.i. & n.i. & -5 & 0.08 & -5 & 0.15 & -7 & 0.09 \\
\hline 155 & $(2 E)$-dodecenyl acetate & 1608 & -13 & 0.11 & -18 & 0.07 & -18 & 0.14 & n.i. & n.i. \\
\hline 156 & 1-hexadecene & 1593 & 4 & 0.01 & n.i. & n.i. & n.i. & n.i. & n.i. & n.i. \\
\hline 157 & guaiol & 1600 & -1 & 0.13 & -5 & 0.12 & -5 & 0.15 & n.i. & n.i. \\
\hline 158 & epiglobulol & 1608 & n.i. & n.i. & -8 & 0.56 & -9 & 0.32 & -11 & 0.17 \\
\hline 159 & humulene epoxide II & 1608 & -1 & 2.59 & -4 & 2.38 & -4 & 0.56 & n.i. & n.i. \\
\hline 160 & humulane-1,6-dien-3-ol & 1619 & n.i. & n.i. & n.i. & n.i. & n.i. & n.i. & -9 & 0.27 \\
\hline 161 & 1,10-di-epi-cubenol & 1618 & -6 & 0.72 & -9 & 0.61 & -9 & 0.75 & n.i. & n.i. \\
\hline 162 & 5-epi-7-epi- $\alpha$-eudesmol & 1607 & 8 & 0.48 & 5 & 0.37 & 5 & 0.32 & n.i. & n.i. \\
\hline 163 & isolongifolan-7- $\alpha$-ol & 1618 & 2 & 0.13 & -1 & 0.05 & -1 & 0.13 & n.i. & n.i. \\
\hline 164 & cis-isolongifolanone & 1612 & n.i. & n.i. & 5 & 0.09 & 5 & 0.03 & n.i. & n.i. \\
\hline 165 & 1-epi-cubenol & 1627 & 0 & 2.49 & -3 & 2.16 & -3 & 1.90 & n.i. & n.i. \\
\hline 166 & $\begin{array}{l}\text { caryophylla-4(12),8(13)-dien- } \\
5-\alpha-o l\end{array}$ & 1639 & -4 & 0.67 & -7 & 0.50 & -7 & 0.40 & n.i. & n.i. \\
\hline 167 & epi- $\alpha$-muurolol & 1640 & 2 & 1.95 & -1 & 1.28 & -1 & 1.48 & -3 & 0.41 \\
\hline 168 & $\alpha$-muurolol & 1644 & 3 & 2.55 & 0 & 2.06 & 0 & 1.82 & n.i. & n.i. \\
\hline 169 & selin-11-en-4- $\alpha$-ol & 1658 & n.i. & n.i. & -8 & 0.91 & -8 & 2.30 & n.i. & n.i. \\
\hline 170 & $\alpha$-cadinol & 1652 & 2 & 1.78 & n.i. & n.i. & n.i. & n.i. & -2 & 0.60 \\
\hline 171 & vulgarone B & 1649 & 5 & 0.04 & 3 & 0.03 & n.i. & n.i. & n.i. & n.i. \\
\hline 172 & $\begin{array}{l}\text { 14-hydroxy-9-epi- }(E) \text { - } \\
\text { caryophyllene }\end{array}$ & 1668 & -11 & 0.25 & -14 & 0.42 & -14 & 0.27 & n.i. & n.i. \\
\hline 173 & (E)-9-tetradecen-1-ol & 1668 & -5 & 0.08 & -8 & 0.06 & n.i. & n.i. & n.i. & n.i. \\
\hline 174 & intermedeol & 1667 & -2 & 0.28 & -5 & 0.22 & -5 & 0.36 & n.i. & n.i. \\
\hline 175 & (Z)- $\alpha$-santalol & 1674 & -4 & 0.96 & -7 & 0.85 & -7 & 0.84 & n.i. & n.i. \\
\hline 176 & guaia-3,10(14)-dien-11-ol & 1676 & 3 & 0.29 & n.i. & n.i. & -2 & 0.20 & n.i. & n.i. \\
\hline 177 & khusinol & 1675 & n.i. & n.i. & 0 & 0.25 & 0 & 0.17 & n.i. & n.i. \\
\hline 178 & eudesma-4(15),7-dien-1- $\beta$-ol & 1687 & -3 & 0.06 & -5 & 0.08 & -5 & 0.34 & n.i. & n.i. \\
\hline 179 & apiole $^{\mathrm{d}, \mathrm{e}}$ & 1677 & 8 & 0.03 & n.i. & n.i. & n.i. & n.i. & n.i. & n.i. \\
\hline 180 & $\alpha$-bisabolol & 1685 & 2 & 0.03 & -1 & 0.02 & n.i. & n.i. & n.i. & n.i. \\
\hline 181 & (Z)- $\alpha$-trans-bergamotol & 1690 & 2 & 0.10 & 0 & 0.05 & n.i. & n.i. & n.i. & n.i. \\
\hline 182 & $(Z, E)$-farnesol & 1713 & -11 & 0.04 & -14 & 0.02 & -14 & 0.03 & n.i. & n.i. \\
\hline
\end{tabular}


Table 2. The list of identified compounds, literature LTPRI values, LTPRI differences and their percentage composition in the essential oils from Piper glabratum analyzed by GC×GC/qMS (cont.)

\begin{tabular}{|c|c|c|c|c|c|c|c|c|c|c|}
\hline \multirow{2}{*}{ No. } & \multirow{2}{*}{ Compound } & \multirow{2}{*}{$\mathrm{LTPRI}_{\mathrm{lit}}{ }^{\mathrm{a}}$} & \multicolumn{2}{|c|}{ Spring } & \multicolumn{2}{|c|}{ Summer } & \multicolumn{2}{|c|}{ Autumn } & \multicolumn{2}{|c|}{ Winter } \\
\hline & & & $\Delta_{\text {LTPRI }}{ }^{b}$ & $\mathrm{~V}^{\mathrm{c}} / \%$ & $\Delta_{\text {LTPRI }}{ }^{\mathrm{b}}$ & $\mathrm{V}^{\mathrm{c}} / \%$ & $\Delta_{\text {LTPRI }}{ }^{\mathrm{b}}$ & $\mathrm{V}^{\mathrm{c}} / \%$ & $\Delta_{\text {LTPRI }}{ }^{\mathrm{b}}$ & $\mathrm{V}^{\mathrm{c}} / \%$ \\
\hline 183 & 14-hydroxy- $\alpha$-humulene & 1713 & -9 & 0.07 & -13 & 0.07 & -9 & 0.11 & n.i. & n.i. \\
\hline 184 & heptadecane & 1700 & n.i. & n.i. & 2 & 0.07 & n.i. & n.i. & n.i. & n.i. \\
\hline 185 & longifolol & 1713 & 4 & 0.07 & 2 & 0.05 & -1 & 0.05 & n.i. & n.i. \\
\hline 186 & (E)-nerolidyl acetate & 1716 & 9 & 0.04 & 8 & 0.02 & n.i. & n.i. & n.i. & n.i. \\
\hline 187 & cedr- $8(15)$-en- 9 - $\alpha$-ol acetate & 1741 & -1 & 0.01 & n.i. & n.i. & n.i. & n.i. & n.i. & n.i. \\
\hline 188 & $\beta$-acoradienol & 1762 & -8 & 0.06 & -10 & 0.04 & n.i. & n.i. & n.i. & n.i. \\
\hline 189 & 14-oxy- $\alpha$-muurolene & 1767 & -5 & 0.03 & n.i. & n.i. & n.i. & n.i. & n.i. & n.i. \\
\hline 190 & 14-hydroxy- $\alpha$-muurolene & 1779 & -4 & 0.04 & -5 & 0.03 & n.i. & n.i. & n.i. & n.i. \\
\hline 191 & ethyl myristate & 1793 & n.i. & n.i. & 4 & 0.08 & n.i. & n.i. & n.i. & n.i. \\
\hline 192 & 14 -hydroxy- $\delta$-cadinene & 1803 & -4 & 0.02 & n.i. & n.i. & n.i. & n.i. & n.i. & n.i. \\
\hline 193 & methyl hexadecanoate & 1921 & 7 & 0.01 & 9 & 0.08 & n.i. & n.i. & n.i. & n.i. \\
\hline 194 & methyl oleate & 2087 & 11 & 0.01 & 13 & 0.03 & n.i. & n.i. & n.i. & n.i. \\
\hline 195 & methyl stearate & 2128 & n.i. & n.i. & 4 & 0.12 & n.i. & n.i. & n.i. & n.i. \\
\hline 196 & tetracosane & 2400 & n.i. & n.i. & 6 & 0.01 & n.i. & n.i. & n.i. & n.i. \\
\hline 197 & pentacosane & 2500 & n.i. & n.i. & 3 & 0.01 & n.i. & n.i. & n.i. & n.i. \\
\hline 198 & hexacosane & 2600 & n.i. & n.i. & 2 & 0.01 & n.i. & n.i. & n.i. & n.i. \\
\hline 199 & heptacosane & 2700 & n.i. & n.i. & 2 & 0.01 & n.i. & n.i. & n.i. & n.i. \\
\hline
\end{tabular}

${ }^{a} \mathrm{LTPRI}_{\mathrm{lit}}$ : linear-temperature-programmed retention index from literature; ${ }^{\mathrm{b}} \Delta_{\mathrm{LTPRI}}$ : LTPRI differences (experimental LTPRI - literature LTPRI); ${ }^{\mathrm{c}} \mathrm{V}$ : percentage of volume of the compounds related to the total of tentatively identified peaks; in bold: ${ }^{\mathrm{d}}$ compounds with $\pi$-conjugated system; ${ }^{\mathrm{e}}$ compounds with aromatic rings. n.i.: not identified.

The essential oil from summer leaves showed the highest number of identified compounds (142), the main representative being the monoterpenes $\beta$-pinene $(9.66 \%)$ and artemisia triene (8.47\%), and oxygenated sesquiterpene $(E)$-nerolidol $(6.76 \%)$. The sesquiterpenes hydrocarbons showed a high relative concentration $(40.50 \%)$, and included compounds such as $\alpha$-selinene $(4.29 \%)$ and $(E)$-caryophyllene $(4.26 \%)$. Notable differences were recorded in the essential oils composition during the season. Indeed, 30 compounds were identified only in essential oil from summer leaves, among them $\beta$-santalene, 3-thujyl acetate, and neryl acetone.

The essential oil from spring leaves showed 136 identified compounds. The major constituents were found to be $(E)$-nerolidol ( $8.45 \%)$, sesquiterpene bicyclogermacrene $(6.93 \%)$, and oxygenated sesquiterpene caryophyllene oxide $(5.56 \%)$. The sesquiterpenes hydrocarbons displayed the highest contribution $(54.10 \%)$ among which (E)-caryophyllene (4.89\%), and $\alpha$-humulene $(4.41 \%)$ were detected at high relative concentrations. A group of 36 compounds were identified only in this sample, among which $(E)$-methyl cinnamate and trans-cadina-1(6),4-diene were the most representative. Phenylpropanoids were also identified exclusively in this oil.

As well as summer, essential oil from autumn leaves presented a high relative concentration of $(E)$-nerolidol
(8.86\%), $\beta$-pinene $(6.75 \%)$, and artemisia triene $(6.40 \%)$. Similar to the oils from summer and spring leaves, the essential oil from autumn leaves presented a high relative concentration of sesquiterpenes hydrocarbons (47.20\%), among which $\alpha$-selinene (6.19\%), germacrene D $(5.47 \%),(E)$-caryophyllene $(4.90 \%)$ were the most representative compounds. This oil showed 101 identified compounds, 7 of which were not identified in the other analyzed oils, such as trans-muurola-3,5-diene and $\alpha$-calacorene.

The essential oil from winter leaves was the poorest analyzed oil, with only 26 compounds identified. It presented germacrene D $(77.40 \%), \delta$-elemene $(4.53 \%)$, and bicyclogermacrene (3.49\%) as its main constituents. Furthermore, it presented the highest relative content of sesquiterpenes hydrocarbons $(98.00 \%)$ of all four oils studied. Two compounds (elixene and humulane-1,6-dien3 -ol ) were found in the oil obtained in winter, which were absent in the other oil analyzed. The typical dry winter in the Dourados region might explain the significant difference in the yield and chemical composition of the essential oil from winter leaves.

In sum, all of the analyzed oils consisted of sesquiterpenes hydrocarbons as a major fraction (Figure 2). The oils from summer and autumn leaves were characterized by higher relative contents of monoterpene hydrocarbons in comparison with the other two samples. Furthermore, the 


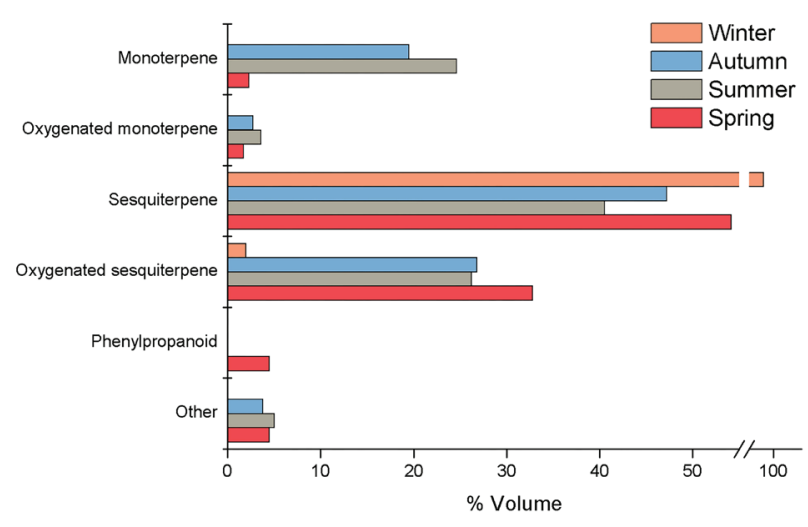

Figure 2. Semi-quantitative distribution of the main classes of compounds in the essential oils of Piper glabratum.

lowest relative amount of oxygenated sesquiterpenes was observed in essential oil from winter leaves.

There are few studies available in the literature regarding the chemical profile of essential oils from P. glabratum. Assis et al. ${ }^{25}$ analyzed the oil using 1D-GC and identified 67 compounds, among them phenylpropanoids as eugenol and apiole. The major compounds were $\beta$-caryophyllene, longiborneol, and (E)-nerolidol. While Branquinho et al. ${ }^{10}$ used the same approach and found 27 compounds, among them $\beta$-pinene, longiborneol, $\alpha$-pinene, and $(E)$-caryophyllene as the major compounds. The majority of the compounds found in those studies were also found in ours; however, the use we made of $\mathrm{GC} \times \mathrm{GC} / \mathrm{qMS}$ has showed the real richness and complexity of these oils, and allowed for its detailed chemical characterization.

\section{Antioxidant and antifungal activities}

\section{DPPH scavenging}

The half maximal inhibitory concentration $\left(\mathrm{IC}_{50}\right)$ values of the essential oils from leaves collected in summer, spring and autumn, and BHT were 28.08 $\pm 0.22,28.12 \pm 0.29$, $33.45 \pm 0.41$, and $17.98 \pm 0.33 \mu \mathrm{g} \mathrm{mL}^{-1}$, respectively. The antioxidant activities of the oils were around $60 \%$ of the antioxidant activity of the synthetic antioxidant BHT.

This is the first study related to the antioxidant activity of essential oil from Piper glabratum. However, the antioxidant activities of the EOs of other Piper species have been documented in the literature. ${ }^{6,26,27}$ There are not many studies on the antioxidant activity of isolated compounds of essential oils, and most of them refer to the activity of the whole matrix. However, the isolated monoterpenes 1,8-cineole, $\beta$-myrcene, sabinene, $\alpha$-terpinene, $\gamma$-terpinene and terpinolene, besides the sesquiterpenes, bisabolol and farnesol, have been shown antioxidant activity in previous studies. ${ }^{28}$ These compounds were identified in the essential oils from $P$. glabratum, suggesting that they might be involved in antioxidant activity observed in the assays.

\section{$\beta$-Carotene bleaching (BCB)}

The antioxidant activity of the essential oils from leaves collected in summer, spring and autumn was 57.40 \pm 0.99 , $60.07 \pm 0.25$, and $66.43 \pm 0.38 \%$ respectively, expressed as percent inhibition relative to the control. The BCB method works in an aqueous emulsion of linoleic acid and $\beta$-carotene, which is discolored by the radicals generated by the spontaneous oxidation of the fatty acid, promoted by thermal induction. ${ }^{29}$

The presence of an antioxidant can be retarded the $\beta$-carotene discoloration by competing with it for the radicals. Therefore, the presence of compounds with similar structure to $\beta$-carotene in the essential oils, such as $\pi$-conjugated molecules, could explain the observed antioxidant activity. As can be seen in Table 2 (compounds in bold), these type of hydrocarbons displayed high contribution-12, 16 and $18 \%$ of the chromatographic area in the essential oils from spring, summer and autumn leaves, respectively-among which $(E)$-methyl cinnamate, $(E, E)-\alpha$-farnesene and artemisia triene were detected at high relative concentrations.

\section{Antifungal activity}

Essential oils from summer, spring and autumn leaves exhibited potent activity against Candida albicans yeast (MIC values of $26 \pm 0.4,27 \pm 0.7$, and $32 \pm 0.2 \mu \mathrm{g} \mathrm{mL}^{-1}$, respectively). The antifungal activity of these essential oils was lower than that of nystatin, the positive control (MIC value of $3.0 \pm 0.2 \mu \mathrm{g} \mathrm{mL} \mathrm{m}^{-1}$ ).

The antimicrobial activity of essential oil has been explained by the presence of monoterpenes and sesquiterpenes with aromatic rings and phenolic hydroxyl groups and other active terpenes, as well as alcohols, aldehydes and esters, all of which might contribute to the overall essential oil antimicrobial effect of the essential oil. ${ }^{30}$ Several aromatic compounds were identified in the P. glabratum essential oils (Table 2). These compounds represented 5.2, 0.7 and $1.3 \%$ of the chromatographic area in the oils from spring, summer and autumn leaves, respectively. Compounds among which $(E)$ and $(Z)$-methyl cinnamate (spring) and trans-calamenene (summer and autumn) were detected at high relative concentrations. Furthermore, some compounds identified in the essential oil from $P$. glabratum, such as $\beta$-pinene and $(E)$-caryophyllene, are known to possess antimicrobial activity against Candida albicans yeast. ${ }^{31,32}$ Thus, these compounds might play an important role in the antifungal findings. 


\section{Conclusions}

In this study, $\mathrm{GC} \times \mathrm{GC} / \mathrm{qMS}$ has proved to be a versatile tool for the analysis of the essential oils from P. glabratum, its use allowing for an in-depth knowledge about the chemical composition of the oils. Due to the variability of the environmental factors, the essential oils obtained from leaves collected at different seasons showed important differences in chemical profile. However, in spite of these differences, the essential oils from spring, summer, and autumn leaves showed similar and strong antioxidant and antifungal activities. The oils from winter leaves were poor in yield and number of identified compounds, and it was not possible at the time to evaluate its activities. These findings point to the influence of the seasonality on the biological activities of this plant.

\section{Acknowledgments}

Authors thank to CAPES (Coordenação de Aperfeiçoamento de Pessoal de Nível Superior) and $\mathrm{CNPq}$ (Conselho Nacional de Desenvolvimento Científico e Tecnológico) for the financial support to this work.

\section{References}

1. de Matos, S. P.; Lucca, L. G.; Koester, L. S.; Talanta 2019, 195 , 204.

2. Bassolé, I. H. N.; Juliani, H. R.; Molecules 2012, 17, 3989.

3. Xiang, C. P.; Shi, Y. N.; Liu, F. F; Li, H. Z.; Zhang, Y. J.; Yang, C. R.; Xu, M.; Nat. Prod. Commun. 2016, 11, 1403.

4. Scott, I. M.; Jensen, H. R.; Philogène, B. J. R.; Arnason, J. T.; Phytochem. Rev. 2008, 7, 65.

5. Perigo, C. V.; Torres, R. B.; Bernacci, L. C.; Guimarães, E. F.; Haber, L. L.; Facanali, R.; Vieira, M. A. R.; Quecini, V.; Marques, M. O. M.; Ind. Crops Prod. 2016, 94, 528.

6. da Silva, J. K. R.; Pinto, L. C.; Burbano, R. M. R.; Montenegro, R. C.; Guimarães, E. F.; Andrade, E.; Maia, J. G. S.; Ind. Crops Prod. 2014, 58, 55.

7. Andrés, M. F.; Rossa, G. E.; Cassel, E.; Vargas, R. M. F.; Santana, O.; Díaz, C. E.; González-Coloma, A.; Food Chem. Toxicol. 2017, 109, 1086.

8. Prando, T. B. L.; Baciquete, T. D. F.; Vieira, J. A. C.; Bressan, J.; Gasparotto, F. M.; Jesus, D. R.; Cardozo Jr., E. L.; LourençO, E. L. B.; Gasparotto Jr., A.; J. Evidence-Based Complementary Altern. Med. 2014, ID 615109.

9. Flores, N.; Jiménez, I. A.; Giménez, A.; Ruiz, G.; Gutiérrez, D.; Bourdy, G.; Bazzocchi, I. L.; J. Nat. Prod. 2008, 71, 1538.

10. Branquinho, L. S.; Santos, J. A.; Cardoso, C. A. L.; Mota, J. S.; Lanza Jr., U.; Kassuya, C. A. L.; Arena, A. C.; J. Ethnopharmacol. 2017, 198, 372.
11. Liu, Z.; Phillips, J.; J. Chromatogr. Sci. 1991, 29, 227.

12. Tranchida, P. Q.; Franchina, F. A.; Mondello, L.; Flavour Fragrance J. 2017, 32, 218.

13. Dimandja, J. M. D.; Stanfill, S. B.; Grainger, J.; Patterson, D. G.; J. High Resolut. Chromatogr. 2000, 23, 208.

14. Mostafa, A.; Edwards, M.; Górecki, T.; J. Chromatogr. A 2012 , 1255, 38 .

15. Lebanov, L.; Tedone, L.; Kaykhaii, M.; Linford, M. R.; Paull, B.; Chromatographia 2019, 82, 357.

16. Silva, B. J. G.; Tranchida, P. Q.; Purcaro, G.; Queiroz, M. E. C.; Mondello, L.; Lanças, F. M.; J. Chromatogr. A 2012, 1255, 177.

17. von Mühlen, C.; Marriott, P. J.; Anal. Bioanal. Chem. 2011, $401,2351$.

18. GCMS Solution, version 4.20; Agilent, Wilmington, USA, 2014.

19. GCImage software, 2.2b1; Zoex Corp., Houston, USA, 2014.

20. Adams, R. P.; Identification of Essential Oil Components by Gas Chromatography/Mass Spectrometry, $4^{\text {th }}$ ed.; Allured: Illinois, USA, 2007.

21. Gupta, D.; Bhardwaj, R.; Gupta, R. K.; Afr. J. Tradit., Complementary Altern. Med. 2011, 8, DOI: 10.4314/ajtcam. v8i4.8.

22. Jayaprakasha, G. K.; Singh, R. P.; Sakariah, K. K.; Food Chem. 2001, 73, 285.

23. Hammer, K. A.; Carson, C. F.; Riley, T. V.; J. Appl. Microbiol. 2003, 95, 853 .

24. Adahchour, M.; Brandt, M.; Baier, H. U.; Vreuls, R. J.; Batenburg, A. M.; Brinkman, U. A.; J. Chromatogr. A 2005, 1067, 245.

25. Assis, A.; Brito, V.; Bittencourt, M.; Silva, L.; Oliveira, F.; Oliveira, R.; J. Essent. Oil Res. 2013, 25, 203.

26. da Silva, J. K. R.; Pinto, L. C.; Burbano, R. M. R.; Montenegro, R. C.; Andrade, E. H. A.; Maia, J. G. S.; Lipids Health Dis. 2016, 15, 174.

27. Bagheri, H.; Yazid, M.; Abdul, B.; Solati, Z.; Talanta 2014, 121, 220.

28. González-Burgos, E.; Gómez-Serranillos, M. P.; Curr. Med. Chem. 2012, 19, 5319.

29. Prieto, M. A.; Rodríguez-Amado, I.; Vázquez, J. A.; Murado, M. A.; J. Agric. Food Chem. 2012, 60, 8983.

30. Belletti, N.; Ndagijimana, M.; Sisto, C.; Guerzoni, M. E.; Lanciotti, R.; Gardini, F.; J. Agric. Food Chem. 2004, 52, 6932.

31. Özer, H.; Sökmen, M.; Güllüce, M.; Adigüzel, A.; Şahin, F.; Sökmen, A.; Kiliç, H.; Bariş, Ö.; J. Agric. Food Chem. 2007, 55, 937.

32. Maggi, F.; Cecchini, C.; Cresci, A.; Coman, M. M.; Tirillini, B.; Sagratini, G.; Papa, F.; Fitoterapia 2009, 80, 68.

Submitted: February 5, 2019 Published online: August 20, 2019 